\title{
Local 5G Operator Architecture for Delay Critical Telehealth Applications
}

\author{
Rakshitha De Silva* $^{*}$ Yushan Siriwardhana ${ }^{\dagger}$, Tharaka Samarasinghe ${ }^{\ddagger}$, Mika Ylianttila ${ }^{\S}$, Madhusanka Liyanage ${ }^{\Uparrow}$ \\ *‡Department of Electronic and Telecommunication Engineering, University of Moratuwa, Sri Lanka. \\ $\dagger \S{ }^{\top}$ Centre for Wireless Communications, University of Oulu, Finland. \\ ${ }^{\ddagger}$ Department of Electrical and Electronic Engineering, University of Melbourne, Australia. \\ ${ }^{\top}$ School of Computer Science, University College Dublin, Ireland. \\ Email: 169221f@uom.lk*, \{firstname.lastname\}@oulu.fił ${ }^{\dagger}$, tharakas@uom.lk, madhusanka@ucd.ie
}

\begin{abstract}
Network softwarization enables the novel concept of Local 5G Operator (L5GO) networks, for deploying localized 5G solutions to serve case and location specific communication needs. This paper proposes a L5GO network architecture for delay critical future telehealth services, considering two use cases on augmented reality assisted and robotic aided surgery. The paper compares the latency performance of the proposed L5GO architecture with a traditional legacy network and a network equipped with Multi-access Edge Computing (MEC). The results highlight the unique advantages of utilizing an L5GO to cater the communication needs of delay critical telehealth, compared to a traditional network.
\end{abstract}

Index Terms-3GPP, 5G, e-health, Local 5G Operator, URLLC.

\section{INTRODUCTION}

Telehealth, which allows access to health care services remotely, is considered to be a key application in next generation wireless networks [1]. The Coronavirus Disease 2019 (COVID-19) pandemic, has made telehealth a focal point, due to the critical requirement of minimizing personal interactions for decelerating the spread of the virus [2]. Furthermore, ensuring the health of the healthcare personnel has become paramount with healthcare facilities operating at their capacity. This paper focuses on delay critical telehealth applications that may have inherent constraints in terms of direct personal interactions, and studies how the fifth generation wireless technology for digital communications, also known as 5G, can be used effectively in such a setting.

$5 \mathrm{G}$ is envisioned to bring about a paradigm shift in communications, providing ultra-low latency, higher bandwidth and massive connectivity. Innovative use cases that stem on the capabilities of $5 \mathrm{G}$ are expected to emerge, with more emphasis on case and location specific communications. Facilities such as hospitals, shopping malls, sports arenas, universities, and factories, already demand for customized wireless connectivity solutions, with varying levels of reliability, data rates, latency and security [3]. Most of these differential Quality-of-Service (QoS) demands are beyond the capabilities of the conventional legacy Mobile Network Operator (MNO) based networks. The focus of traditional MNOs is providing generic mobile communication services to mass geographical areas such as countries or provinces. The investment cycles of MNOs are generally long with a high infrastructure cost. Therefore, additional capital and operational expenditure, and increased effort, prevent the deployment of case and location specific network services by MNOs.

Multi-access Edge Computing (MEC) brings the computing capabilities to the edge of the network, enabling latency aspects in 5G, while supporting high bandwidth. As MEC operates closer to the location of the applications, real-time altering of application aware performance optimization is possible. This is useful in medical applications that need low latency. However, most MEC configurations will fail to provide Ultra Reliability and Low Latency Communication (URLLC), which is mandatory for many telehealth use cases. These stringent QoS demands have made way to new players such as Local 5G Operators (L5GOs), to enter the market with an aim of providing case and location specific communication services [4], [5].

The intention of L5GOs is to deploy localized communication services to satisfy the context-aware extreme service requirements for a selected group of users. Even though the L5GOs serve confined locations, they need to collaborate with the 5G MNOs, thus the L5GO deployments must follow the standards defined by standardization organizations such as the 3rd Generation Partnership Project (3GPP)[3] [6]. In addition, the $\mathrm{L} 5 \mathrm{GO}$ should lease spectrum from a regulator or an MNO, in the form of local spectrum licenses. The L5GO may maintain its own infrastructure or lease the infrastructure owned by an infrastructure vendor. The L5GO can provide roaming services to subscribers of other MNOs, upon the their arrival to the L5GO coverage area, given that an agreement is in place between the two parties.

Due to the communication demands of a L5GO network being highly variable, a tailored custom architecture that is ready to serve user needs is preferred over a universal network architecture. This paper presents a modified architecture for an emerging L5GO network, which provides ultra-low latency communication services suited for telehealth. The study is based on two such use cases that require URLLC defined by 3GPP. They are, Augmented Reality (AR) assisted surgery and robotic aided surgery. The proposed architecture comprises of both the core and the access 5G network functions needed to support communication.

The paper compares the latency performance of the pro- 
posed L5GO architecture with a traditional MNO network and a network equipped with MEC. The paper highlights the unique advantages of utilizing an L5GO to cater the communication needs of the two use cases of interest. We also present the modifications that should be made to the MNO or the MEC networks to enable them satisfy such delay critical use cases. We show that the required upgrades have serious concerns in terms of financial feasibility, to further highlight the utility of the proposed L5GO architecture for delay critical telehealth applications.

The remainder of the paper is organized as follows: Section II presents the two delay critical telehealth use cases. Section III describes the L5GO network registration process and the Protocol Data Unit (PDU) session establishment, and presents the proposed L5GO architecture. Section IV presents three network deployment models, which are compared in Section $\mathrm{V}$ through numerical results. Section VI concludes the paper.

\section{USE CASES}

The study considers two use cases in telehealth that have stringent latency constraints, thus requiring a network with ultra-low latency. Moreover, the use cases involve the delivery of critical care in a healthcare facility, where the medical team and the patients are not in close proximity (no physical contact). Both use cases require $99.9999 \%$ communication service availability [7].

\section{A. Augmented Reality Assisted Surgery}

3D imaging methods such as Magnetic Resonance (MR) and Computer Tomography (CT) scans are already being used to assist doctors in diagnostics. Image guided surgery is gaining popularity in the healthcare industry with the increasing penetration of technologies like AR. Although there are several ways of generating AR views to identify and follow the exact 3D position of a human body [7], this study considers only a generic scenario where AR can be used in assisting surgeries.

To this end, let us consider a scenario where AR images are generated by a device at the patient's end, and transferred to an image processing server for augmentation. The augmented images are then transferred to a viewing console at the surgeon's physical location. The high-quality images that necessitate complex processing create latency in the communication process. A Grand Master Clock (GMC) is used to synchronize the entire setup.

\section{B. Robotic Aided Surgery}

Robotic aided surgery is important in invasive surgical procedures such as delicate tissue replacements and in situations where accessibility to internal organs is difficult. In a robotic aided surgery, the patient's end is equipped with complex instruments that replicate the surgeon's hand movements. These instruments can perform high precision manoeuvres. The control console at the surgeon's end is fed with haptic feedback, to create the experience of a real surgery [7].

All equipment including the surgeon's console are synchronized with the GMC with an accuracy of $1 \mu \mathrm{s}$. Robotic Aided
Kidney Transplant (RAKT) is a typical surgery performed using this method. Such a surgery requires the transfer of 240 images per second over the network. Having two repetitive errors in any direction is fatal, as they may result in an incorrect command being sent to the actuators. By taking this into account, 3GPP specifies the message error rate to be lower than $0.0001 \%$, which sets the minimum requirement in terms of reliability, for a network utilized for a RAKT type surgical procedure [7].

\section{The L5GO Network Architecture}

This section proposes a possible L5GO network architectural model that facilitates delay critical $5 \mathrm{G}$ telehealth applications. The architectural model is set up to support the communication requirements of the two use cases presented in Section II. The network registration, which handles the registration of the terminal devices in the $5 \mathrm{G}$ network, and PDU session establishment procedures, which creates a data session between two hosts in the $5 \mathrm{G}$ network, are set based on the 3GPP specifications [8].

\section{A. The L5GO Network Registration}

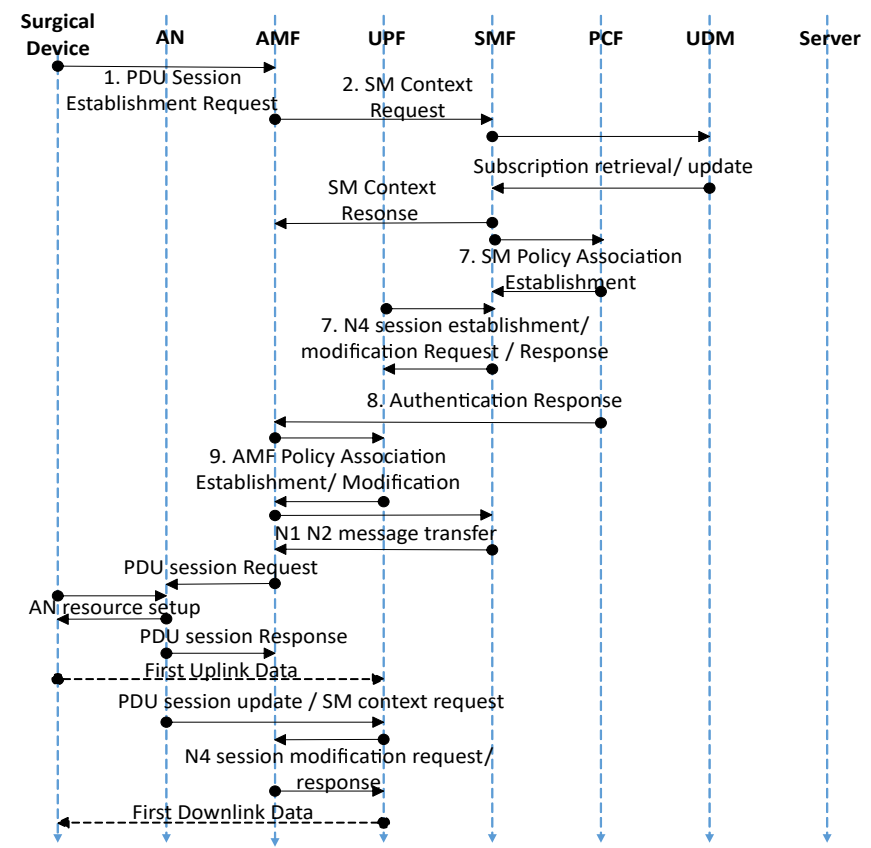

Fig. 1: The message flow diagram for device registration.

The console sends the registration request via the L5GO Access Network (AN) to the next generation NodeB (gNB), and generates a registration request at the Access and Mobility Management Function (AMF). As an L5GO typically serves a location specific small user group, it is unlikely to have multiple AMFs in the network, thus eliminating the AMF selection procedure. The AMF exchanges identity request messages with the console in order to establish a connection. Then, the AMF validates the device through the Equipment Identity Register (EIR), and communicates with the Authentication Server Function (AUSF) for device authentication. The AUSF 
authenticates the device after communicating with the Unified Data Management (UDM) and retrieving the authentication data. The AUSF completes the authentication process with a response to the AMF. Upon successful authentication, the AMF contacts the Policy Control function (PCF) for the respective policy association for the console. Then, the AMF sends an update to the Session Management Function (SMF) to notify the session context. The AMF generating and sending a registration accept message to the console follows. The console replies with a registration complete message to the AMF, and completes the registration process [8].

\section{B. The Protocol Data Unit Session Establishment}

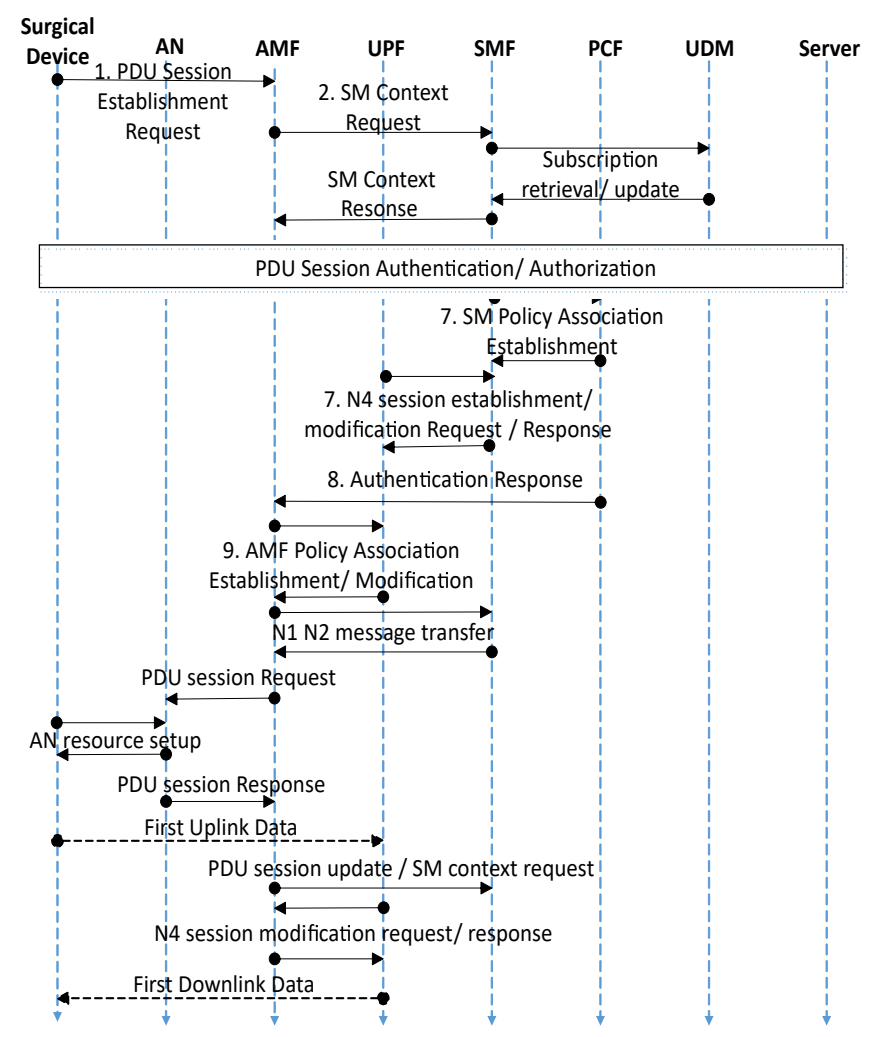

Fig. 2: The message flow diagram for the PDU session establishment.

Upon successful registration, the console initiates the PDU session establishment procedure with the image processing server, according to the 3GPP specifications [8]. Firstly, the PDU session establishment request by the console is forwarded to the AMF via the L5GO gNB. The AMF generates and sends a PDU session create/session management context request to the SMF. The SMF interacts with the UDM for subscription retrieval, and updates related attributes for this particular session. Then, the SMF responds to the AMF with a session management context. Afterwards, the PDU session authentication/authorization takes place through message exchanges between the NFs in the surgical device and the imaging server, as shown in Fig. 2. The SMF coordinates with the PCF for policy association for the session. The SMF and the UPF exchange session establishment/modification requests and responses. Then, an authentication response is sent to the AMF by the PCF. The AMF policy association establishment/modification messages interchange between the AMF and the UPF follows. Next, the SMF and the AMF initiate a message transfer session. The AMF transfers the PDU session ID details to the gNB so that the gNB can coordinate with the surgical device for the gNB defined resource setup. Based on that, the L5GO's AN sends a PDU session response to the AMF, and the AMF sends a PDU session update/session management context request to the SMF. The SMF then sends a request message to the UPF for session modification. Upon retrieval of this message by the UPF, the SMF transmits the response of the PDU session update to the AMF, which concludes the PDU session establishment process [8].

\section{The Proposed Architecture}

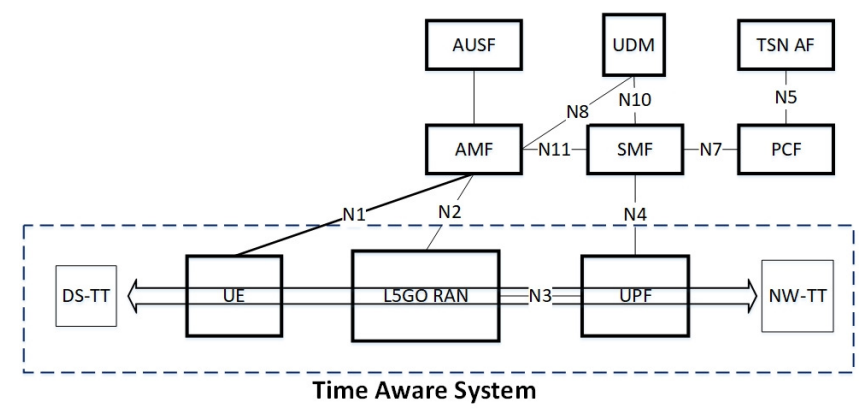

Fig. 3: The proposed network architecture.

The proposed L5GO network architecture for delay critical telehealth applications is illustrated in Fig. 3. It uses the Time Sensitive Network (TSN) logical bridge architecture defined by 3GPP [9] as the base, together with the IEEE 802.1AS time aware system. The IEEE 802.1 TSN enables transmission of various types of critical data over a bridged Ethernet network. Transmitted data can be of different Quality of Service (QoS) levels, regardless of the low latency end-to-end connectivity provided by the TSN [10].

The TSN bridge is between the Device-side TSN translator (DS-TT) and Network-side TSN translator (NW-TT) [9]. When operating the $5 \mathrm{G}$ core network elements, the RAN and the communication links are invisible to the TSN bridge because it works as an end-to-end tunnel. The entire system is synchronized by a GMC. The Time Sensitive Network Application Function (TSN AF) establishes the end-to-end tunnel connectivity and maintains it throughout the session, whereas the rest of the $5 \mathrm{G}$ network functions serve their original purpose.

A 5G network equipped with the TSN bridge features is equipped to deliver ultra-low latency services to the subscribers. As the use cases of interest are for surgeries of stationary organs, their End-to-End (E2E) delay requirement is considered to be below $18 \mathrm{~ms}$ [7]. However, when it comes to cases with moving organs, the delay threshold decreases drastically. 


\section{The Network Deployment Models}

The study considers three possible network deployment models;

- L5GO network

- Traditional MNO

- MEC enabled MNO network.

The image generating devices, the image processing server and the surgical devices are located inside the hospital premises in all three models. The study only focuses on the communication process associated with the data transfer, as it is the most critical stage with regards to ultra-low latency. This means, the delays associated with the device registration and the PDU session establishment are neglected. During the data transfer process, the generated data gets transferred to the image processing server via the access network and the core network function UPF. The image processing server augments the required information and sends the augmented information to the AR consoles, with the involvement of the UPF.

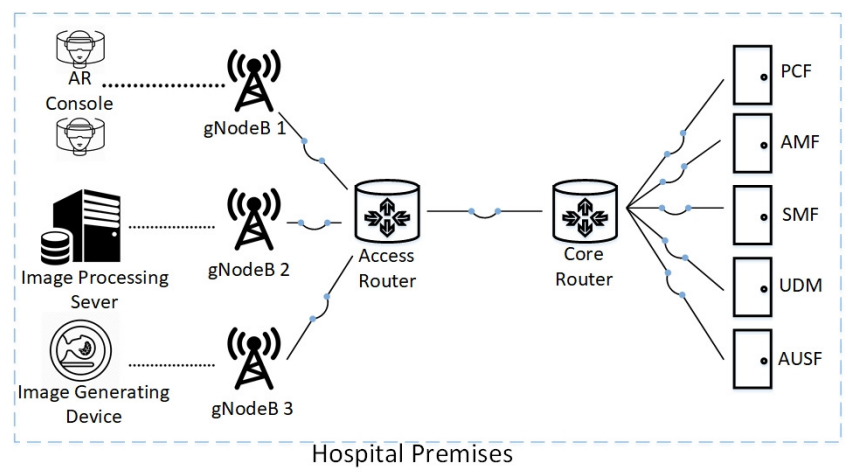

Fig. 4: Deployment model for a L5GO serving a hospital premises

In the L5GO deployment model, a single L5GO provides connectivity to the hospital premises, through a multitude of gNBs with small coverage areas. The communication requirements of the surgical devices, the image processing server and the monitor consoles are catered through different gNBs, as illustrated in Fig. 4. Most importantly, the L5GO core network is also located inside the hospital premises.

In the MNO deployment model, the MNO provides connectivity to all communicating devices in the hospital premises through its $5 \mathrm{G}$ network. Fig. 5 depicts the MNO deployment model. The core network of the MNO resides outside the hospital facility. In a practical setting, there can be $N$ such healthcare facilities located in a geographically distributed manner, and served by the same MNO.

The third deployment model, which is the MEC network, has a similar access network as the MNO model, but the core network functions are located at the edge, thus located closer to the hospital premises. The study assumes that $M$ healthcare facilities with the similar setup are served by the MEC network, similar to the MNO model. We assume $M \leq N$.

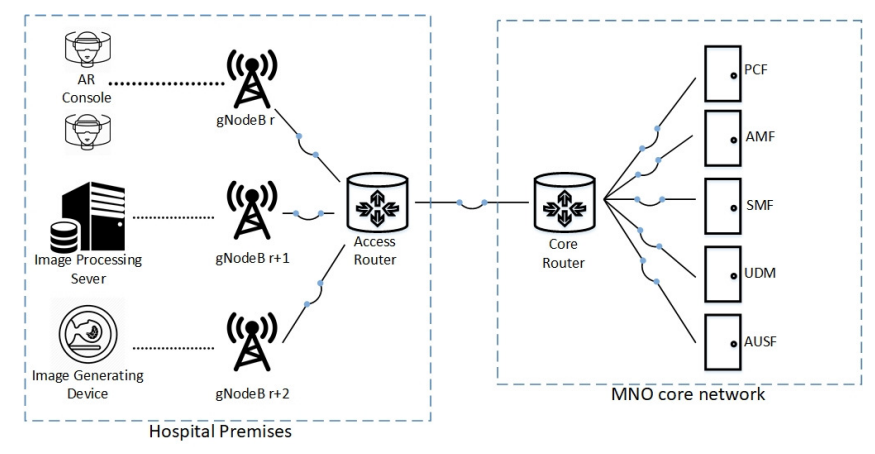

Fig. 5: Deployment model for a MNO serving a hospital premises

(a)

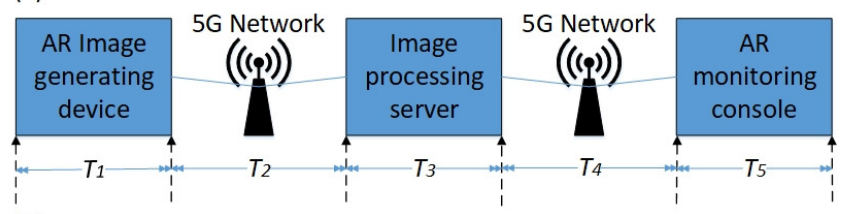

(b)

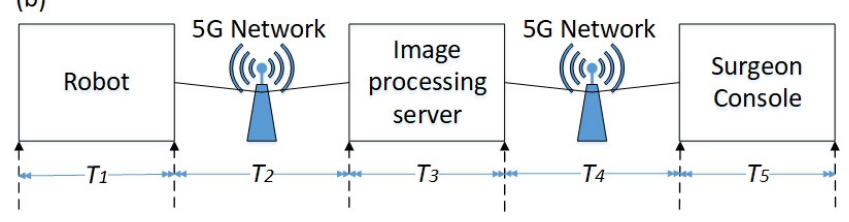

Fig. 6: (a) Latency breakdown for use case II-A

(b) Latency breakdown for use case II-B

The different latency components of the two use cases are illustrated in Fig. 6. We assume Frequency Division Duplexing (FDD), and a $120 \mathrm{kHz}, 7 \mathrm{~s}$ mini-slot bandwidth for the access network deployment. Moreover, we assume a fiber backbone for the backhaul links to support high data rates and achieve ultra-low latency. Table I tabulates general calculation parameters considered for the two use cases.

TABLE I: General calculation parameters

\begin{tabular}{|c|c|}
\hline Parameter & Value \\
\hline Latency between the terminal devices and the AN $T_{A N}$ & $0.33 \mathrm{~ms}[11]$ \\
Latency between the AN and the core network $T_{B H}$ & $0.05 \mathrm{~ms}$ per km [12] \\
Mean service time of a gNB $T_{N B}$ & $0.1 \mathrm{~ms}$ \\
Mean service time of the access router $T_{A R}$ & $0.1 \mathrm{~ms}$ \\
Mean service time of the core router $T_{C R}$ & $0.05 \mathrm{~ms}$ \\
Mean service time of the 5G NF $T_{N} F$ & $0.01 \mathrm{~ms}$ \\
Maximum acceptable AR imaging system latency & $14 \mathrm{~ms}[7]$ \\
Maximum acceptable Robotic Aided Surgery system latency & $20 \mathrm{~ms} \mathrm{[7]}$ \\
Distance to the L5GO core network & $100 \mathrm{~m}[7]$ \\
Healthcare facilities served by the MNO $(N)$ & 10 \\
Healthcare facilities served by the MEC $(M)$ & 3 \\
\hline
\end{tabular}

\section{RESUlts AND Discussion}

\section{A. The Impact of the Distance to the Core Network}

The E2E latency of the system comprises of the image generation and processing delay, the operating time of the surgical devices and the latency of the communication infrastructure as illustrated in Fig. 6. The human reaction time is neglected. 
According to the latency breakdown in Fig. 6, the total E2E latency is given by

$$
T_{\text {lat }}=\sum_{r=1}^{5} T_{r},
$$

where $T_{r}$ denotes the latency of the $r$-th stage, and $r \in$ $\{1, \ldots, 5\}$. For AR assisted surgery, the threshold value for $T_{\text {lat }}$ is $14 \mathrm{~ms}$ [7], given that the surgery is performed on static organs, where the only moving object is the surgeon's hand. If the surgery is performed on a moving organ such as the heart, the threshold for $T_{l a t}$ should be lower than $14 \mathrm{~ms}$ to achieve sufficient precision. Further, it is known that $T_{1}+T_{3}+T_{5}=11$ $\mathrm{ms}$ for AR assisted surgery use cases [7]. The tolerable latency threshold for robotic aided surgery is slightly higher at 20 ms. This threshold also decreases when moving organs are considered. For robotic aided surgery, $T_{1}+T_{3}+T_{5}=16 \mathrm{~ms}$ [7]. The latency in the $5 \mathrm{G}$ network can be calculated by

$$
T_{2}=T_{4}=T_{A N}+T_{B H}+T_{N B}+T_{A R}+T_{C R}+T_{N F},
$$

where the symbols are defined in Table I.

The E2E latency values for various network settings are presented in Fig. 7 and Fig. 8, for AR assisted surgery and robotic aided surgery, respectively. In particular, the figures show how the latency changes with the distance to the core network. For both use cases, three MEC core network distances $(10 \mathrm{~km}, 25$ $\mathrm{km}$ and $50 \mathrm{~km}$ ) are considered. All these three settings will not satisfy the required average latency threshold for AR assisted surgery, whereas for robotic aided surgery, the setting with a distance of $10 \mathrm{~km}$ to the core network satisfies the latency constraint. We can observe that for the MNO network setting to work, the distance to the core network should be brought down to $100 \mathrm{~m}$. Any distance greater than this violates the latency constraint. Finally, we can observe that the L5GO best suits the two use cases.

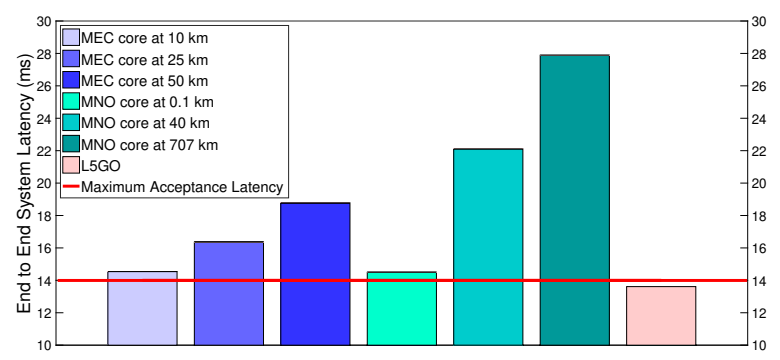

Fig. 7: E2E latency of use case II-A with respect to distance to core network

\section{B. Impact of the Network Function Processing Delay}

The Network Function (NF) processing delay $T_{N F}$ is a crucial component in E2E latency. The NF processing delay mainly depends on the operator resources and the network traffic load. To this end, we have

$$
T_{N F} \propto \frac{\text { network load }}{\text { operator resources }} \text {. }
$$

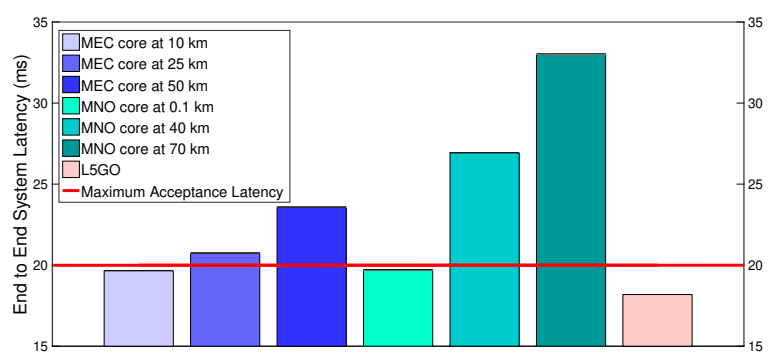

Fig. 8: E2E latency of use case II-B with respect to distance to core network

Therefore, the latency values related to the $5 \mathrm{G}$ network can be written as

$$
\begin{aligned}
T_{2}=T_{4}=T_{A N}+T_{B H}+ & T_{N B}+T_{A R}+T_{C R} \\
& +K . \frac{\text { network load }}{\text { operator resources }},
\end{aligned}
$$

where $K=1$ according to the proposed architecture in Section III.

An MNO typically possess higher computational and network resources compared to an L5GO, whereas an MEC possess more resources compared to an L5GO, but comparatively less resources compared to an MNO. Since the NF processing delay is inversely proportional to the operator resources, we consider four different resource levels for the MNO and the MEC networks as outlined in Table II, where $J$ is the resource multiplier. The total resources for an MNO or an MEC with a resource multiplier $J$, can be written as $J \times R_{L 5 G O}$, where $R_{L 5 G O}$ denotes the total resources in the L5GO network. We check whether tolerable latency is achievable with the MNO and the MEC networks by increasing their resource levels compared to the L5GO.

TABLE II: Increased resource levels at the MNO and the MEC networks compared to the L5GO

\begin{tabular}{|c|c|}
\hline Resource Level & Resource Multiplier for the MNO/MEC \\
\hline$L_{1}$ & $J=1$ \\
$L_{2}$ & $J=10$ \\
$L_{3}$ & $J=100$ \\
$L_{4}$ & $J=1000$ \\
\hline
\end{tabular}

We consider values of $0.01 \mathrm{~ms}$ and $0.05 \mathrm{~ms}$ for the $T_{N F}$ of the L5GO. Note that an MNO has $N=10$ L5GOs in the network, and an MEC has $M=3$ L5GOs in the network. Thus, it is not hard to see that the $T_{N F}$ of an MNO can be calculated by multiplying the $T_{N F}$ of the L5GO network by $\frac{N}{R}$. Similarly, the $T_{N F}$ of an MEC can be calculated by multiplying the $T_{N F}$ of the L5GO network by a factor $\frac{M}{R}$. Then, we calculate the maximum distance that the networks with the additional resources can have to their core networks, that is the length of the fiber backhaul. These values are tabulated in Table III for a $T_{N F}$ of $0.01 \mathrm{~ms}$ at the L5GO, and in Table IV for a $T_{N F}$ of $0.05 \mathrm{~ms}$ at the L5GO. The length of the backhaul is denoted by $D_{B H}$. 
It is clear that the length of the backhaul, that is, where the core network can be deployed, varies based on the resource availability. It is possible for the MNO to support the required E2E latency, under all four resource levels. This is due to the underlying reason that the increased resource levels lead to a lower $T_{N F}$ value at the MNO. However, it is interesting to consider the distance values to the core network although the delay constraints are satisfied. Obviously, the distance to the core network monotonically increases with the network resources at the MNO. However, it can be noted that the gradient of this increasing slope is rather small, thus the gain is not significant compared to the additional costs. As an example, considering the AR assisted surgery use case, for $J=100, D_{B H}$ is $2.2859 \mathrm{~km}$, and for $J=1000 D_{B H}$ is $2.40985 \mathrm{~km}$. The gain is approximately $120 \mathrm{~m}$, which does not justify a 100-fold resource upgrade. It can also be seen that the backhaul delay $T_{B H}$ is more prominent compared to the NF processing delay $T_{N F}$ for the two use cases of interest.

Since the MEC only serves 3 health facilities simultaneously, it shows a higher gain in terms of $D_{B H}$ compared to the MNO for similar resource levels. The MEC too satisfies the required latency thresholds when the resource levels are increased. For Robotic aided surgery, $D_{B H}$ is $7.76625 \mathrm{~km}$ for $J=10$, and $D_{B H}$ is $7.8557 \mathrm{~km}$ for $J=100$. Similar to the MNO, the change is insignificant when taking the cost of resource upgrade (increase of $90 \mathrm{~m}$ from 10-fold resource upgrade). At $T_{N F}=0.01 \mathrm{~ms}$, the gain is about $170 \mathrm{~m}$ for AR assisted surgery, for the same 10-fold resource upgrade.

TABLE III: Distance to core network when L5GO $T_{N F}=0.01$ $\mathrm{ms}$ for use case II-A and II-B

\begin{tabular}{|l|l|l|l|l|l|l|}
\hline \multirow{2}{*}{ Level } & \multicolumn{3}{|c|}{ MNO } & \multicolumn{3}{c|}{ MEC } \\
\cline { 2 - 7 } & $\begin{array}{l}T_{N F} \\
(\mathrm{~ms})\end{array}$ & $\begin{array}{l}\mathrm{AR} \\
D_{B H}(\mathrm{~km})\end{array}$ & $\begin{array}{l}\text { Robotic } \\
D_{B H}(\mathrm{~km})\end{array}$ & $\begin{array}{l}T_{N F} \\
(\mathrm{~ms})\end{array}$ & $\begin{array}{l}\text { AR } \\
D_{B H}(\mathrm{~km})\end{array}$ & $\begin{array}{l}\text { Robotic } \\
D_{B H}(\mathrm{~km})\end{array}$ \\
\hline$L_{1}$ & 0.1 & 1.8024 & 7.1372 & 0.03 & 2.32045 & 7.76625 \\
$L_{2}$ & 0.01 & 1.95065 & 7.1907 & 0.003 & 4.9829 & 7.8557 \\
$L_{3}$ & 0.001 & 2.2859 & 7.3603 & 0.0003 & 2.7495 & 7.9232 \\
$L_{4}$ & 0.0001 & 2.40985 & 7.57005 & 0.00003 & 2.91955 & 8.07075 \\
\hline
\end{tabular}

TABLE IV: Distance to core network when L5GO $T_{N F}=$ $0.05 \mathrm{~ms}$ for use case II-A and II-B

\begin{tabular}{|l|l|l|l|l|l|l|}
\hline \multirow{2}{*}{ Level } & \multicolumn{3}{|c|}{ MNO } & \multicolumn{3}{c|}{ MEC } \\
\cline { 2 - 7 } & $\begin{array}{l}T_{N F} \\
(\mathrm{~ms})\end{array}$ & $\begin{array}{l}\text { AR } \\
D_{B H}(\mathrm{~km})\end{array}$ & $\begin{array}{l}\text { Robotic } \\
D_{B H}(\mathrm{~km})\end{array}$ & $\begin{array}{l}T_{N F} \\
(\mathrm{~ms})\end{array}$ & $\begin{array}{l}\text { AR } \\
D_{B H}(\mathrm{~km})\end{array}$ & $\begin{array}{l}\text { Robotic } \\
D_{B H}(\mathrm{~km})\end{array}$ \\
\hline$L_{1}$ & 0.5 & 0.67635 & 6.5351 & 0.15 & 1.47935 & 5.78545 \\
$L_{2}$ & 0.05 & 1.7324 & 6.6696 & 0.015 & 1.99245 & 6.04125 \\
$L_{3}$ & 0.005 & 1.806 & 7.29235 & 0.0015 & 2.126 & 7.6696 \\
$L_{4}$ & 0.0005 & 2.0823 & 7.5262 & 0.00015 & 2.2269 & 8.26105 \\
\hline
\end{tabular}

Overall, our results show that increasing resources at the MNO and the MEC networks is not a financially feasible investment for delay critical telehealth applications. The results also further highlight the importance of the proposed L5GO architecture for delay critical telehealth applications.

\section{CONCLUSIONS}

This paper has proposed a Local 5G Operator (L5GO) architecture for delay critical telehealth applications, considering two delay critical healthcare use cases, i.e., Augmented Reality (AR) assisted surgery and robotic aided surgery. The proposed architecture has been designed based on the 5G Time Sensitive Network (TSN) architecture. The paper has highlighted the performance gains from the proposed L5GO network compared to the conventional Mobile Network Operator (MNO) based networks, and the Multi-access Edge Computing (MEC) based MNO networks, through an End-to-End (E2E) latency study. Numerical results have shown that the proposed L5GO architecture satisfies the stringent latency constraints of both use cases. On the other hand, the MNO or the MEC based network architectures fail to satisfy these constraints. It has also been shown that if it is required to use the MNO or the MEC networks for such delay critical use cases, the distance to the core network should be considerably decreased, and the total network resource levels should be considerably increased. The upgrade deems infeasible financially, which further highlights the utility of the proposed L5GO architecture for delay critical telehealth applications.

\section{ACKNOWLEDGEMENT}

This work is supported by Academy of Finland in 6Genesis Flagship (grant no. 318927) and 5GEAR projects, and European Union in RESPONSE 5G (Grant No: 789658) project.

\section{REFERENCES}

[1] E. Liu, E. Effiok, J. Hitchcock, "Survey on health care applications in 5G networks", IET Communications, vol. 14, no. 7, pp. 1073-1080, Apr. 2020.

[2] D. Soldani, "Fighting COVID-19 with 5G enabled technologies". White paper, Huawei Technologies. Apr. 2020.

[3] Y. Siriwardhana, P. Porambage, M. Liyanage, J. S. Waliay, M. Matinmikko-Blue, M. Ylianttila, "Micro-operator driven local 5G network architecture for industrial internet," in. proc. IEEE Wireless Communications and Networking Conference, Marrakech, Morocco, Apr. 2019

[4] S. Kekki et al., "MEC in 5G networks," ETSI White Paper No. 28, First edition, Jun. 2018

[5] T. Taleb, K. Samdanis, B. Mada, H. Flinck, S. Dutta, D. Sabella, ”On Multi-Access edge computing: A Survey of the emerging 5G network edge cloud architecture and orchestration," IEEE Communications Surveys \& Tutorial , Vol. 19, No. 3, pp 1667-1681, May 2017

[6] M. Matinmikko, M. Latva-aho , P. Ahokangas, S. Yrjola, T. Koivumaki, " Micro operators to boost local service delivery in 5G," Wireless Personal Communications, pp 69-82, May 2017

[7] 3GPP, "3GPP TR 22.826 ; Study on communication services for critical medical applications (Release 17)," Technical report, Aug. 2019. [Online]. Available:https://portal.3gpp.org/desktopmodules/ Specifications/SpecificationDetails.aspx?specificationId $=3546$

[8] 3GPP, "3GPP TS 23.502 ; System Aspects;System architecture for the 5G System (5GS);Stage 2 (Release 16)," Technical specification, Dec. 2019, [Online]. Available:https://portal.3gpp.org/desktopmodules/ Specifications/SpecificationDetails.aspx?specificationId=3546

[9] 3GPP, "3GPP TS 23.501 ;System architecture for the 5G system (5GS);Stage 2 (Release 16)," technical specification, Dec. 2019, [Online]. Available:https://portal.3gpp.org/desktopmodules/ Specifications/SpecificationDetails.aspx? specificationId $=3546$

[10] J.Farkas, L.L Bello, C. Gunther, "Time-sensitive networking standards", IEEE Communications Standards Magazine, Jun. 2018

[11] J. Sachs, G. Wikström, T. Dudda, R. Baldemair, K. Kittichokechai, "5G radio network design for ultra-reliable low-latency communication," IEEE Network, vol. 32, No. 2, Mar./Apr. 2018

[12] M. Jaber, M. A. Imran, R. Tafazolli, A. Tukmanov, "5G backhaul challenges and emerging research directions: A survey," IEEE Access vol. 4, pp. 1743-1766, Apr. 2016. 